\title{
Exacerbation of diabetic neuropathy can mimic SARS-CoV-2 associated pure dysautonomic Guillain-Barre syndrome
}

\author{
Josef Finsterer ${ }^{1}$ (D)
}

Received: 9 August 2021 / Revised: 9 August 2021 / Accepted: 27 January 2022 / Published online: 25 February 2022

(c) Journal of NeuroVirology, Inc. 2022

To the Editor,

With interest we read the article by Biassoni et al. about a 79-year-old male who developed pure dysautonomic Guillain-Barre syndrome (GBS) 10 days after onset of moderate COVID-19 (Biassoni et al. 2021). Dysautonomic manifestations included urinary retention and orthostatic dysfunction (Biassoni et al. 2021). The patient had a previous history of diabetes and arterial hypertension (Biassoni et al. 2021). We have the following comments and concerns.

A limitation of the study is that information about diabetes in the index patient is missing. We should know the onset of diabetes, quality of diabetes control, medication, number of hypoglycaemic or hyperglycaemic episodes, and if there were any indications for diabetic neuropathy prior to onset of COVID-19. Missing in this respect are the HbA1c values, results of previous nerve conduction studies (NCSs), and ophthalmologic investigations. It is unclear how exacerbation of diabetic neuropathy due to the viral infection was excluded.

We do not agree with the classification of GBS as pure dysautonomic. Since nerve conduction velocity of motor nerves was reduced, there was at least electrophysiological involvement of motor nerves.

Missing are systematic investigations for autonomic dysfunction. We should be told if there was REM sleep behaviour disorder, reduced heart rate variability, oversensitivity to light, renal dysfunction, bowel dysfunction, impotentia, anaemia, syncope, left ventricular hypertrophy, arterial hypertension, postprandial hypotension, constipation, or hyper- or hypohidrosis. Assuming that there was indeed pure dysautonomic GBS, why should only be the urinary bladder and the orthostatic reaction be involved and not the entire

Josef Finsterer

fifigs1@yahoo.de

1 Neurology \& Neurophysiology Center, Postfach 20,

1180 Vienna, Austria spectrum of autonomic functions? One would expect that more autonomic functions were impaired.

We should be informed why the patient received insulin during hospitalisation. Obviously, diabetes worsened during the viral infection, why it is conceivable that autonomic dysfunction was rather due to worsening diabetic neuropathy than GBS.

GBS is usually diagnosed according to the Brighton criteria. However, when applying these criteria, level 1 of certainty was not reached, further suggesting that there was diabetic neuropathy rather than GBS.

Missing are follow-up investigations, to assess if intravenous immunoglobulins truly exhibited a beneficial effect. Particularly missing are electrophysiological follow-up investigations to assess if neuropathy improved with clinical improvement.

Overall, this interesting case report has limitations which should be addressed before attributing partial autonomic dysfunction to SARS-CoV-2 associated GBS. In a patient with diabetes, which worsened during the infection with SARS-CoV-2, autonomic dysfunction is rather attributable to exacerbating diabetic neuropathy than GBS.

Author contribution JF: design, literature search, discussion, first draft, critical comments, final approval.

\section{Declarations}

Ethics approval The statement of ethics was in accordance with ethical guidelines.

Informed consent Not applicable.

Conflict of interest The author declares no competing interests.

Disclaimer The study was approved by the institutional review board. 


\section{Reference}

Biassoni E, Assini A, Gandoglia I, Benedetti L, Boni S, Pontali E, Feasi M, Gandolfo F, Del Sette M (2021) The importance of thinking about Guillain-Barré syndrome during the COVID-19 pandemic: a case with pure dysautonomic presentation. J Neurovirol 2:1-4. https://doi.org/10.1007/s13365-021-00997-7

Publisher's Note Springer Nature remains neutral with regard to jurisdictional claims in published maps and institutional affiliations. 